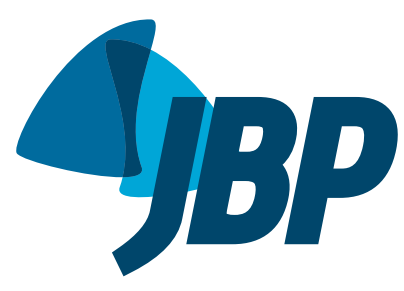

1. Sleep - Laboratório de Estudo dos Distúrbios do Sono, Centro Médico BarraShopping, Rio de Janeiro (RJ) Brasil.

2. Instituto de Doenças do Tórax Universidade Federal do Rio de Janeiro, Rio de Janeiro (RJ) Brasil.

3. Programa de Pós-Graduação em Ciências da Saúde, Universidade Federal de Goiás, Goiânia (GO) Brasil.

4. Hospital Geral de Goiânia Dr. Alberto Rassi, Goiânia (GO) Brasil.

5. Clínica do Aparelho Respiratório, Goiânia (GO) Brasil.

Submitted: 25 May 2017

Accepted: 3 September 2017.

Study carried out at Sleep - Laboratório de Estudo dos Distúrbios do Sono, Centro Médico BarraShopping, Rio de Janeiro (RJ) Brasil and at the Clínica do Aparelho Respiratório, Goiânia (GO) Brasil.

\section{Validation of the STOP-Bang questionnaire as a means of screening for obstructive sleep apnea in adults in Brazil}

\author{
Ricardo Luiz de Menezes Duarte ${ }^{1,2}$, Lorena Barbosa de Moraes Fonseca ${ }^{3,4,5}$, \\ Flavio José Magalhães-da-Silveira', Erika Aparecida da Silveira ${ }^{3}$, \\ Marcelo Fouad Rabahi3 $3,4,5$
}

\begin{abstract}
Objective: To validate the Portuguese-language version of the STOP-Bang (acronym for Snoring, Tiredness, Observed apnea, high blood Pressure, Body mass index, Age, Neck circumference, and Gender) questionnaire, culturally adapted for use in Brazil, as a means of screening for obstructive sleep apnea (OSA) in adults. Methods: In this validation study, we enrolled patients $\geq 18$ years of age, recruited between May of 2015 and November of 2016. All patients completed the STOP-Bang questionnaire and underwent overnight polysomnography. To evaluate the performance of the questionnaire, we used contingency tables and areas under the (receiver operating characteristic) curve (AUCs). Results: We included 456 patients. The mean age was $43.7 \pm 12.5$ years, and 291 $(63.8 \%)$ of the patients were male. On the basis of the apnea-hypopnea index (AHI), we categorized OSA as mild/moderate/severe (any OSA; $\mathrm{AHI} \geq 5$ events/h), moderate/ severe $(A H I \geq 15$ events $/ h$ ), or severe (AHI $\geq 30$ events/h). The overall prevalence of OSA was $78.3 \%$, compared with $52.0 \%$, and $28.5 \%$ for moderate/severe and severe OSA, respectively. The most common score on the STOP-Bang questionnaire was 4 points (n $=106)$, followed by 3 points $(n=85)$ and 5 points $(n=82)$. An increase in the score was paralleled by a reduction in sensitivity with a corresponding increase in specificity for all AHI cut-off points. The AUCs obtained for the identification of any, moderate/severe, and severe OSA were: $0.743,0.731$, and 0.779 , respectively. For any OSA, the score on the questionnaire (cut-off, $\geq 3$ points) presented sensitivity, specificity, and accuracy of $83.5 \%, 45.5 \%$, and $75.2 \%$, respectively. Conclusions: The STOP-Bang questionnaire performed adequately for OSA screening, indicating that it could be used as an effective screening tool for the disorder.
\end{abstract}

Keywords: Sleep apnea, obstructive/diagnosis; Polysomnography; Diagnostic techniques and procedures; Surveys and questionnaires.

\section{INTRODUCTION}

Obstructive sleep apnea (OSA) is the most common sleep disorder and a major public health problem, affecting $2-4 \%$ of the adult population. ${ }^{(1,2)}$ However, due to the aging of the population ${ }^{(3)}$ and the obesity epidemic, ${ }^{(4)}$ the actual prevalence of OSA might be higher than previously reported. ${ }^{(5-7)}$ The signs, symptoms, and consequences of OSA are a direct result of the disturbances associated with repetitive upper airway collapse ${ }^{(8)}$ : sleep fragmentation, hypoxemia, hypercapnia, marked oscillations in intrathoracic pressure, and increased sympathetic activity. Left untreated, OSA limits the ability to perform activities of daily living, worsens quality of life, compromises personal safety, and decreases labor productivity, as well as increasing health care expenditures. ${ }^{(3,4)}$

The current gold standard for the diagnosis of OSA is overnight polysomnography in a laboratory. However, OSA has become so prevalent that the available sleep laboratories have been overwhelmed. Sleep laboratories around the world have long waiting lists of patients suspected of having OSA. (9) To address this issue, several screening questionnaires and clinical screening models have been developed to help identify patients with suspected OSA. ${ }^{(10-16)}$ The great majority of these models were developed in other countries, and their reproducibility in Brazil remains unclear. The use of practical screening tools will probably translate to a higher rate of diagnosis and a reduction in costs. That is especially true for portable diagnostic methods in areas with limited resources.

The STOP-Bang (acronym for Snoring, Tiredness, Observed apnea, high blood Pressure, Body mass index, Age, Neck circumference, and Gender) questionnaire and its predecessor, the STOP questionnaire, were first developed and validated for use in surgical patients. ${ }^{(14)}$ The STOP and STOP-Bang are both self-report questionnaires and consist of 4 and 8 yes/no questions, respectively. When a cut-off score of $\geq 3$ points was used in a sample of surgical patients, the STOP-Bang questionnaire showed the following conditional probabilities for the diagnosis

Correspondence to:

Ricardo Luiz de Menezes Duarte. Sleep - Laboratório de Estudo dos Distúrbios do Sono, Centro Médico BarraShopping, Avenida das Américas, 4666, sala 309,

Barra da Tijuca, CEP 22649-900, Rio de Janeiro, RJ, Brasil.

Tel.: 5521 2430-9222. Fax: 5521 2430-9220. E-mail: rlmduarte@gmail.com

Financial support: None. 
of OSA ${ }^{(14)}$ : sensitivity of $83.6 \%$, specificity of $56.4 \%$, a positive predictive value (PPV) of $81.0 \%$, and a negative predictive value (NPV) of $60.8 \%$.

The population of Brazil includes individuals from diverse ethnic, racial, and social groups. Given that the Brazilian population is racially and ethnically diverse, it could be useful to determine the validity and performance of the STOP-Bang questionnaire in this setting. To our knowledge, there have been no studies determining the reproducibility of this questionnaire in Brazil. The objective of the present study was to validate the Portuguese-language version of the STOP-Bang questionnaire that has been culturally adapted for use in Brazil( ${ }^{(17)}$ in patients with suspected OSA who were submitted to overnight polysomnography.

\section{METHODS}

\section{Patient selection}

Consecutive outpatients were recruited from among those referred for overnight polysomnography at two sleep laboratories in Brazil: one in the city of Goiânia (enrollment from May 2015 to August 2015, n = 229) and one in the city of Rio de Janeiro (enrollment from October 2016 to November 2016, n = 227). The study protocol was approved by the Research Ethics Committees of the Alberto Rassi Hospital, in Goiânia (Protocol no. 752/14), and of the Federal University of Rio de Janeiro, in Rio de Janeiro (Protocol no. $1,764,165)$. All procedures were performed in accordance with the Declaration of Helsinki, and all participating patients gave written informed consent.

All clinical and polysomnography data were collected prospectively. The following inclusion criteria were applied: being $\geq 18$ years of age; not having previously been diagnosed with OSA; and having been referred to a sleep laboratory for polysomnography, for any reason. We excluded patients who had participated in the cultural adaptation of the STOP-Bang questionnaire, ${ }^{(17)}$ as well as those who did not complete the questionnaire fully or correctly and those is whom the polysomnography was incomplete or technically inadequate.

\section{Application of the STOP-Bang questionnaire}

After signing the consent form, the patients completed the STOP-Bang questionnaire. The four initial questions-those corresponding to the "STOP" portion of the questionnaire-were answered by the patients themselves. The responses to the questions corresponding to the "Bang" portion of the questionnaire were collected by the researcher on a standardized form. Body weight was measured in kilograms, and height was measured in meters. The body mass index (BMI) was then calculated as follows: weight in kilograms divided by height in meters squared ( $\mathrm{kg} /$ $\mathrm{m}^{2}$ ). Neck circumference was measured with a 150$\mathrm{cm}$ tape measure, whose smallest markings were at $0.01-\mathrm{cm}$ intervals, and was determined at the level of the cricothyroid membrane.

\section{Sleep studies}

All sleep studies were conducted either in Goiânia, on an Alice 5 diagnostic sleep system (Philips Respironics, Murrysville, PA, USA), or in Rio de Janeiro, on an EMBLA S7000 digital system (Embla Systems Inc., Broomfield, CO, USA). The following were performed: electroencephalography; left and right electro-oculography; electromyography of the submental and anterior tibialis muscles; snore detection; nasal airflow monitoring (with a nasal cannula); respiratory effort assessment (with the use of thoracic and abdominal straps); pulse oximetry; electrocardiography; body position monitoring; and digital video capture. Polysomnography records were scored manually and were interpreted in accordance with existing guidelines, ${ }^{(18)}$ which define apnea as a $\geq$ $90 \%$ reduction in airflow for $\geq 10 \mathrm{~s}$ and hypopnea as a $\geq 30 \%$ reduction in airflow for $\geq 10 \mathrm{~s}$, accompanied by desaturation $\geq 3 \%$ or an arousal. The apnea-hypopnea index (AHI) was calculated by determining the total number of apnea and hypopnea events per hour of sleep. The diagnosis of OSA was based on an AHI $\geq 5$ events/h. The severity of OSA was classified as follows: mild/moderate/severe (any OSA; AHI $\geq 5$ events/h); moderate/severe (AHI $\geq 15$ events/h); or severe (AHI $\geq 30$ events/h). At both sleep laboratories, the physicians who carried out the polysomnography examinations were blinded to the STOP-Bang scores.

\section{Statistical analysis}

Statistical analysis was performed with the IBM SPSS Statistics software package, version 23.0 for Windows (IBM Corporation, Armonk, NY, USA). Continuous variables are expressed as mean and standard deviation, whereas categorical (dichotomous) variables are expressed as absolute and relative frequencies. Groups were compared with the chi-square-test (for dichotomous variables), Student's t-test and one-way ANOVA (for normally distributed continuous variables), or the Mann-Whitney U test and the Kruskal-Wallis test (for non-normally distributed continuous variables). Correlations were evaluated by determining Spearman's correlation coefficient $\left(r_{s}\right)$. The ROC curves and the areas under the curve (AUCs) were assessed at all three AHI thresholds (5 events/h, 15 events/h, and 30 events/h). Multivariate tests were used in order to calculate the odds ratios and their respective $95 \%$ confidence intervals. Using $2 \times 2$ contingency tables, we calculated the following conditional probabilities: sensitivity, specificity, PPV, NPV, accuracy, likelihood ratios, and odds ratios. The post-test probability of each STOP-Bang score was calculated by logistic regression. All statistical tests were two-sided, and values of $p<$ 0.05 were considered statistically significant.

\section{RESULTS}

A total of 522 patients were referred for diagnostic polysomnography. Of those 522 patients, 66 (12.6\%) were excluded, as follows: 50 patients, because the polysomnography was technically inadequate; 
11 patients, because the STOP-Bang questionnaire was completed incorrectly; and 5 patients, because they had previously participated in the cross-cultural adaptation of the questionnaire. Therefore, the final study population comprised 456 patients, in two independent samples: one from Rio de Janeiro $(n=$ 229 ) and one from Goiânia ( $n=227)$. As can be seen in Table $1,63.8 \%$ of the patients were male, the mean age was $43.7 \pm 12.5$ years, the mean BMI was 32.1 $\pm 7.8 \mathrm{~kg} / \mathrm{m}^{2}$, and the mean neck circumference was $40.8 \pm 4.3 \mathrm{~cm}$. We observed statistically significant differences between the patients with and without OSA for all continuous variables (age, BMI, and neck circumference), those with OSA (AHI $\geq 5$ events/h) being older ( $p<0.001)$, having a higher BMI $(p=0.001)$, and having a larger neck circumference $(p<0.001)$. Six of the eight items on the STOP-Bang questionnaire presented a statistically significant difference between the patients with and without OSA, the two exceptions being tiredness $(p=0.730)$ and BMI $>35 \mathrm{~kg} / \mathrm{m}^{2}(p$ $=0.705)$. In comparison with the patients without OSA, those with OSA were more likely to snore ( $p=$ $0.002)$, to present with apnea ( $p<0.001)$, to have hypertension ( $p<0.001)$, to be over 50 years of age $(p=0.011)$, to have a neck circumference $>40 \mathrm{~cm}$ $(p<0.001)$, and to be male ( $p<0.001)$. Among the patients with severe OSA ( $\mathrm{AHI} \geq 30$ events/h), the mean age was $45.9 \pm 12.8$ years, the mean BMI was $33.5 \pm 7.9 \mathrm{~kg} / \mathrm{m}^{2}$, and the mean neck circumference was $43.1 \pm 4.2 \mathrm{~cm}$.

The overall prevalence of OSA was $78.3 \%$, compared with $52.0 \%$ and $28.5 \%$ for moderate/severe OSA and severe OSA, respectively. In addition, the overall prevalence of OSA was higher in males than in females ( $84.5 \%$ vs. $67.3 \%)$, as was that of moderate/severe OSA (60.1\% vs. $37.6 \%)$ and severe OSA (36.4\% vs. $14.5 \%)$, the differences being statistically significant ( $p<0.001$ for all). That suggests that male gender influences the occurrence of OSA.
Table 2 shows the adjusted odds ratios for all eight STOP-Bang items in relation to OSA severity. Only three items were independent predictors at all three AHI thresholds: observed apnea, age, and neck circumference. In contrast, the tiredness item was not an independent predictor at any AHI threshold ( $p$ $=0.912$ for any OSA, $p=0.397$ for moderate/severe OSA, and $p=0.097$ for severe OSA). The best predictor of moderate/severe OSA was neck circumference (adjusted OR: 2.347; 95\% CI: 1.445-3.816), followed by age (adjusted OR: 2.132; 95\% CI: 1.308-3.472) and observed apnea (adjusted OR: $1.897 ; 95 \% \mathrm{CI}$ : 1.233-2.923).

Table 3 shows the distribution of the parameters according to OSA severity. The most common STOP-Bang score (occurring in 106 patients) was 4 points, followed by 3 points (occurring in 85 ) and 5 points (occurring in 82). Using the cut-off STOP-Bang score of 3 points, we classified $34.1 \%$ of the patients as having no OSA ( $\mathrm{AHI}<5$ events $/ \mathrm{h}$ ) and $9.4 \%$ as having severe OSA (AHI $\geq 30$ events/h). The mean STOP-Bang score was $3.8 \pm 1.6$ points in the study population as a whole and was significantly lower in females than in males $(2.8 \pm 1.3$ points vs. $4.4 \pm 1.4$ points; $p<0.001)$. In addition, the mean STOP-Bang score increased significantly in parallel with increasing severity of OSA-from $2.8 \pm 1.4$ points for the patients without OSA to $4.1 \pm 1.5$ points for those with any OSA, 4.5 \pm 1.5 points for those with moderate/severe OSA, and $5.0 \pm 1.3$ points for those with severe OSA-the $\mathrm{p}$-value for trend being $<0.001$.

The performance of the STOP-Bang questionnaire is shown in Table 4. As can be seen in the table, the cut-off STOP-Bang score ( $\geq 3$ points) showed the following conditional probabilities for the identification of any OSA: sensitivity of $83.5 \%$, specificity of $45.5 \%$, PPV of $84.7 \%$, NPV of $43.3 \%$, and accuracy of $75.2 \%$. For the identification of moderate/severe OSA, the same STOP-Bang cut-off score had a sensitivity of $88.6 \%$, a

Table 1. Baseline characteristics of the patients screened for obstructive sleep apnea. ${ }^{a}$

\begin{tabular}{|c|c|c|c|c|}
\hline Characteristic & $\begin{array}{l}\text { All patients } \\
\text { (n = 456) }\end{array}$ & $\begin{array}{c}\text { Without OSA } \\
\text { AHI }<5 \text { events } / \mathrm{h} \\
\text { (n = 99) }\end{array}$ & $\begin{array}{c}\text { With OSA } \\
\text { AHI } \geq 5 \text { events/h } \\
(n=357)\end{array}$ & p-value \\
\hline Age (years), mean \pm SD & $43.7 \pm 12.5$ & $36.4 \pm 12.4$ & $44.9 \pm 12.2$ & $<0.001$ \\
\hline BMI $\left(\mathrm{kg} / \mathrm{m}^{2}\right)$, mean $\pm \mathrm{SD}$ & $32.1 \pm 7.8$ & $29.6 \pm 7.0$ & $32.7 \pm 7.9$ & 0.001 \\
\hline $\mathrm{NC}(\mathrm{cm})$, mean $\pm \mathrm{SD}$ & $40.8 \pm 4.3$ & $38.1 \pm 3.8$ & $41.6 \pm 4.2$ & $<0.001$ \\
\hline $\begin{array}{l}\text { AHI (events } / \mathrm{h}) \text {, mean } \pm \text { SD } \\
\text { STOP-Bang items }\end{array}$ & $24.6 \pm 25.2$ & $2.1 \pm 1.4$ & $30.9 \pm 25.1$ & $<0.001$ \\
\hline Snoring (loud) & $313(68.6)$ & $55(55.6)$ & $258(72.3)$ & 0.002 \\
\hline Tiredness & $270(59.2)$ & $57(57.6)$ & $213(59.7)$ & 0.730 \\
\hline Observed apnea & $216(47.4)$ & $29(29.3)$ & $187(52.4)$ & $<0.001$ \\
\hline Pressure (hypertension) & $160(35.1)$ & $20(20.2)$ & $140(39.2)$ & $<0.001$ \\
\hline $\mathrm{BMI}\left(>35 \mathrm{~kg} / \mathrm{m}^{2}\right)$ & $129(28.3)$ & $26(26.3)$ & $103(28.9)$ & 0.705 \\
\hline Age (> 50 years) & $151(33.1)$ & $22(22.2)$ & $129(36.1)$ & 0.011 \\
\hline $\mathrm{NC}(>40 \mathrm{~cm})$ & $236(51.8)$ & $24(24.2)$ & $212(59.4)$ & $<0.001$ \\
\hline Gender (male) & $291(63.8)$ & $45(45.5)$ & $246(68.9)$ & $<0.001$ \\
\hline
\end{tabular}

OSA: obstructive sleep apnea; BMI: body mass index; NC: neck circumference; and AHI: apnea-hypopnea index. ${ }^{a}$ Data are presented as $\mathrm{n}(\%)$, except where otherwise indicated. 
Table 2. Adjusted odds ratios obtained by multivariate analysis for each item of the Portuguese-language version of the STOP-Bang questionnaire. ${ }^{a}$

\begin{tabular}{|c|c|c|c|c|c|c|}
\hline \multirow[t]{4}{*}{ STOP-Bang item } & \multicolumn{6}{|c|}{ OSA severity } \\
\hline & Any & p & Moderate/severe & p & Severe & p \\
\hline & AHI $\geq 5$ events $/ h$ & & $\begin{array}{c}\text { AHI } \geq 15 \\
\text { events/h }\end{array}$ & & $\begin{array}{c}\mathrm{AHI} \geq 30 \\
\text { events/h }\end{array}$ & \\
\hline & $(n=357)$ & & $(n=237)$ & & $(n=130)$ & \\
\hline Snoring (loud) & $1.420(0.851-2.369)$ & 0.179 & $1.666(1.063-2.610)$ & 0.026 & $1.706(0.964-3.021)$ & 0.066 \\
\hline Tiredness & $0.971(0.585-1.612)$ & 0.912 & $1.199(0.787-1.828)$ & 0.397 & $1.515(0.926-2.475)$ & 0.097 \\
\hline Observed apnea & $2.044(1.182-3.546)$ & 0.010 & $1.897(1.233-2.923)$ & 0.004 & $4.016(2.415-6.622)$ & $<0.001$ \\
\hline Pressure (hypertension) & $1.680(0.911-3.095)$ & 0.096 & $1.650(1.039-2.617)$ & 0.034 & 1.745 (1.047-2.915) & 0.033 \\
\hline BMI $\left(>35 \mathrm{~kg} / \mathrm{m}^{2}\right)$ & $1.364(0.711-2.617)$ & 0.350 & $1.222(0.718-2.079)$ & 0.458 & $2.008(1.103-3.649)$ & 0.023 \\
\hline Age (> 50 years) & $2.227(1.193-4.166)$ & 0.012 & $2.132(1.308-3.472)$ & 0.002 & $2.016(1.184-3.436)$ & 0.010 \\
\hline $\mathrm{NC}(>40 \mathrm{~cm})$ & $3.134(1.712-5.747)$ & $<0.001$ & $2.347(1.445-3.816)$ & 0.001 & $1.869(1.055-3.311)$ & 0.032 \\
\hline Gender (male) & $1.663(0.934-2.958)$ & 0.083 & $1.644(0.993-2.724)$ & 0.053 & $2.392(1.265-4.524)$ & 0.007 \\
\hline
\end{tabular}

OSA: obstructive sleep apnea; AHI: apnea-hypopnea index; BMI: body mass index; and NC: neck circumference. avalues are presented as adjusted OR $(95 \% \mathrm{CI})$.

Table 3. Distribution of scores by apnea-hypopnea index $(n=456) .^{\text {a }}$

\begin{tabular}{|c|c|c|c|c|}
\hline \multirow{2}{*}{$\begin{array}{c}\text { STOP-Bang } \\
\text { score }\end{array}$} & Without OSA & \multicolumn{3}{|c|}{ OSA severity } \\
\hline & $\begin{array}{c}\text { AHI }<5 \text { events } / \mathrm{h} \\
\text { (n }=99)\end{array}$ & $\begin{array}{c}\text { Any } \\
\text { AHI } \geq 5 \text { events/h } \\
(n=357)\end{array}$ & $\begin{array}{c}\text { Moderate/severe } \\
\text { AHI } \geq 15 \text { events/h } \\
(n=237)\end{array}$ & $\begin{array}{c}\text { Severe } \\
\text { AHI } \geq 30 \text { events/h } \\
(n=130)\end{array}$ \\
\hline $0(n=2)$ & $1(50.0)$ & $1(50.0)$ & $0(0.0)$ & $0(0.0)$ \\
\hline $1(n=31)$ & $17(54.8)$ & $14(45.2)$ & $7(22.6)$ & $0(0.0)$ \\
\hline $2(n=71)$ & $27(38.0)$ & $44(62.0)$ & $20(28.2)$ & $6(8.5)$ \\
\hline $3(n=85)$ & $29(34.1)$ & $56(65.9)$ & $28(32.9)$ & $8(9.4)$ \\
\hline $4(n=106)$ & $14(13.2)$ & $92(86.8)$ & $62(58.5)$ & $32(30.2)$ \\
\hline $5(n=82)$ & $5(6.1)$ & 77 (93.9) & $57(69.5)$ & $37(45.1)$ \\
\hline $6(n=53)$ & $4(7.5)$ & $49(92.5)$ & 41 (77.4) & $28(52.8)$ \\
\hline $7(n=22)$ & $1(4.5)$ & 21 (95.5) & $19(86.4)$ & $16(72.7)$ \\
\hline $8(n=4)$ & $1(25.0)$ & $3(75.0)$ & $3(75.0)$ & $3(75.0)$ \\
\hline
\end{tabular}

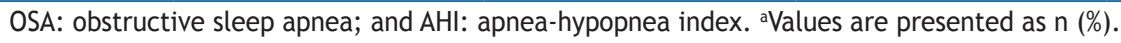

specificity of $35.2 \%$, a PPV of $59.7 \%$, an NPV of $74.0 \%$, and an accuracy of $62.9 \%$; for the identification of severe OSA, it had a sensitivity of $95.4 \%$, a specificity of $30.1 \%$, a PPV of $35.2 \%$, an NPV of $94.2 \%$, and an accuracy of $48.7 \%$. The STOP-Bang score correlated positively with the AHI $\left(r_{s}=0.516 ; p<0.001\right)$. According to the ROC curves (Figure 1), the STOP-Bang questionnaire showed the following AUCs: 0.743 (95\% CI: $0.689-0.798)$ for the diagnosis of any OSA; 0.731 (95\% CI: $0.685-0.777$ ) for the diagnosis of moderate/ severe OSA; and 0.779 (95\% CI: 0.735-0.824) for the diagnosis of severe OSA. Table 5 summarizes the prediction of any OSA, moderate/severe OSA, and severe OSA in relation to the various possible scores obtained on the STOP-Bang questionnaire. For a score $\geq 3$ points, the post-test probability of any OSA, moderate/severe OSA, and severe OSA was $84.7 \%$, $59.6 \%$, and $35.2 \%$, respectively.

\section{DISCUSSION}

In this prospective study, we determined that the STOP-Bang questionnaire shows promise as a screening tool for OSA in patients referred to sleep laboratories in Brazil. The use of the STOP-Bang questionnaire could provide various benefits: reducing the risk of peri- and post-operative complications, which often go undiagnosed in surgical patients with $\mathrm{OSA}^{(19)}$; reducing the occurrence of comorbidities, thus minimizing the health care costs associated with the disorder; and providing a portable diagnostic method, which could be critical in areas with limited resources, where polysomnography is not widely available. In addition, the performance of the STOP-Bang questionnaire was quite similar to that reported in previous studies for the STOP and STOP-Bang questionnaires ${ }^{(14,20-25)}$; that is, high sensitivity and low-to-moderate specificity, which could be especially critical in the more severe forms of OSA, leading to a decrease in accuracy (the proportion of correctly screened individuals). This aspect of its predictive performance (low specificity) has also been reported in various studies designed to translate, adapt, or validate the STOP-Bang questionnaire. ${ }^{(21-25)}$

Our study showed a high prevalence of OSA overall, as well as a high prevalence of moderate/severe OSA and severe OSA. That could be explained by the fact that it was conducted in a sleep laboratory setting. Unlike studies conducted in the community, sleep laboratory studies have reported OSA prevalence rates of $42-76 \%$. $^{(10)}$ Our study, similar to previous studies, demonstrated that the prevalence of OSA 
Table 4. Conditional probabilities of the various possible scores on the Portuguese-language version of the STOP-Bang questionnaire for the prediction of obstructive sleep apnea, by degree of severity $(n=456)$. $^{\text {a }}$

\begin{tabular}{|c|c|c|c|c|c|}
\hline OSA severity & Sensitivity & Specificity & PPV & NPV & Accuracy \\
\hline \multicolumn{6}{|l|}{$\mathrm{AHI} \geq 5$ events $/ \mathrm{h}$} \\
\hline$\geq 1$ vs. $<1$ & 99.7 (99.5-100.0) & $1.0(0.1-2.0)$ & 78.4 (78.2-78.6) & $50.0(2.7-97.3)$ & 78.3 (77.9-78.7) \\
\hline$\geq 2$ vs. $<2$ & $95.8(94.2-97.3)$ & $18.2(12.5-23.6)$ & $80.9(79.5-82.1)$ & $54.5(37.4-70.8)$ & $78.9(76.5-81.3)$ \\
\hline$\geq 3$ vs. $<3$ & $83.5(81.1-85.8)$ & $45.5(37.0-53.8)$ & $84.7(82.3-87.0)$ & $43.3(35.2-51.2)$ & $75.2(71.5-78.8)$ \\
\hline$\geq 4$ vs. $<4$ & $67.8(65.4-69.8)$ & $74.7(66.0-82.2)$ & $90.6(87.4-93.4)$ & $39.2(34.6-43.0)$ & $69.3(65.5-72.5)$ \\
\hline$\geq 5$ vs. $<5$ & $42.0(39.9-43.4)$ & 88.9 (81.3-93.9) & $93.2(88.5-96.3)$ & $29.8(27.3-31.5)$ & $52.2(48.9-54.4)$ \\
\hline$\geq 6$ vs. $<6$ & $20.4(18.7-21.4)$ & 93.9 (87.5-97.5) & $92.4(84.3-96.8)$ & $24.7(23.0-25.6)$ & $36.4(33.6-37.9)$ \\
\hline$\geq 7$ vs. $<7$ & 6.7 (5.4-7.2) & $98.0(93.2-99.6)$ & 92.3 (74.1-98.7) & $22.6(21.5-22.9)$ & $26.5(24.5-27.3)$ \\
\hline 8 vs. $<8$ & $0.8(0.2-1.1)$ & 99.0 (96.9-99.9) & $75.0(22.1-98.7)$ & $21.7(21.2-21.9)$ & $22.1(21.2-22.6)$ \\
\hline \multicolumn{6}{|l|}{$\mathrm{AHI} \geq 15$ events $/ \mathrm{h}$} \\
\hline$\geq 1$ vs. $<1$ & $100.0(99.3-100.0)$ & $0.9(0.2-0.9)$ & $52.2(51.8-52.2)$ & $100.0(19.8-$ & 52.4 \\
\hline$\geq 2$ vs. $<2$ & $97.0(94.6-98.6)$ & $11.9(9.2-13.6)$ & $54.4(53.0-55.3)$ & $78.8(61.2-90.3)$ & $56.1(53.6-57.8)$ \\
\hline$\geq 3$ vs. $<3$ & $88.6(84.8-91.8)$ & $35.2(31.1-38.6)$ & $59.7(57.1-61.8)$ & $74.0(65.5-81.4)$ & $62.9(59.0-66.3)$ \\
\hline$\geq 4$ vs. $<4$ & $76.8(72.4-80.8)$ & $61.2(56.5-65.5)$ & $68.2(64.3-71.7)$ & $70.9(65.4-75.9)$ & $69.3(64.8-73.5)$ \\
\hline$\geq 5$ vs. $<5$ & $50.6(46.4-54.4)$ & $81.3(76.7-85.4)$ & $74.5(68.3-80.1)$ & $60.3(56.9-63.4)$ & $65.4(61.0-69.3)$ \\
\hline$\geq 6$ vs. $<6$ & $26.6(23.3-29.1)$ & 92.7 (89.1-95.4) & $79.7(69.8-87.4)$ & $53.8(51.8-55.4)$ & $58.3(54.9-61.0)$ \\
\hline$\geq 7$ vs. $<7$ & $9.3(7.1-10.4)$ & $98.2(95.8-99.4)$ & $84.6(64.8-94.9)$ & $50.0(48.8-50.6)$ & $52.0(49.7-53.1)$ \\
\hline 8 vs. $<8$ & $1.3(0.4-1.7)$ & $99.5(98.6-100.0)$ & $75.0(22.0-98.7)$ & $48.2(47.8-48.4)$ & $48.5(47.5-48.9)$ \\
\hline \multicolumn{6}{|l|}{$\mathrm{AHI} \geq 30$ events $/ \mathrm{h}$} \\
\hline$\geq 1$ vs. $<1$ & $100.0(98.8-100.0)$ & $0.6(0.1-0.6)$ & $28.6(28.3-28.6)$ & $100.0(19.8-100.0)$ & $28.9(28.2-28.9)$ \\
\hline$\geq 2$ vs. $<2$ & $100.0(96.8-100.0)$ & $10.1(8.8-10.1)$ & 30.7 (29.7-30.7) & $100.0(87.4-100.0)$ & 35.7 (33.9-35.7) \\
\hline$\geq 3$ vs. $<3$ & $95.4(90.3-98.1)$ & $30.1(28.0-31.1)$ & $35.2(33.4-36.2)$ & $94.2(87.9-97.6)$ & $48.7(45.8-50.2)$ \\
\hline$\geq 4$ vs. $<4$ & $89.2(83.0-93.6)$ & $53.7(51.2-55.4)$ & $43.4(40.4-45.6)$ & $92.6(88.3-95.6)$ & $63.8(60.3-66.3)$ \\
\hline$\geq 5$ vs. $<5$ & $64.6(57.3-71.4)$ & 76.4 (73.5-79.1) & $52.2(46.3-57.6)$ & $84.4(81.2-87.4)$ & $73.0(68.8-76.9)$ \\
\hline$\geq 6$ vs. $<6$ & $36.2(29.9-42.0)$ & 90.2 (87.7-92.5) & $59.5(49.1-69.1)$ & $78.0(75.8-80.0)$ & $74.8(71.2-78.1)$ \\
\hline$\geq 7$ vs. $<7$ & $14.6(10.5-17.5)$ & $97.9(96.2-99.0)$ & $73.1(52.7-87.5)$ & $74.2(73.0-75.1)$ & $74.1(71.8-75.8)$ \\
\hline 8 vs. $<8$ & $2.3(0.7-3.0)$ & $99.7(99.0-100.0)$ & $75.0(22.0-98.7)$ & $71.9(71.4-72.1)$ & $71.9(71.0-72.3)$ \\
\hline
\end{tabular}

OSA: obstructive sleep apnea; PPV: positive predictive value; NPV: negative predictive value; and AHI: apneahypopnea index. ${ }^{a}$ Data are presented as estimated value $(95 \% \mathrm{CI})$.
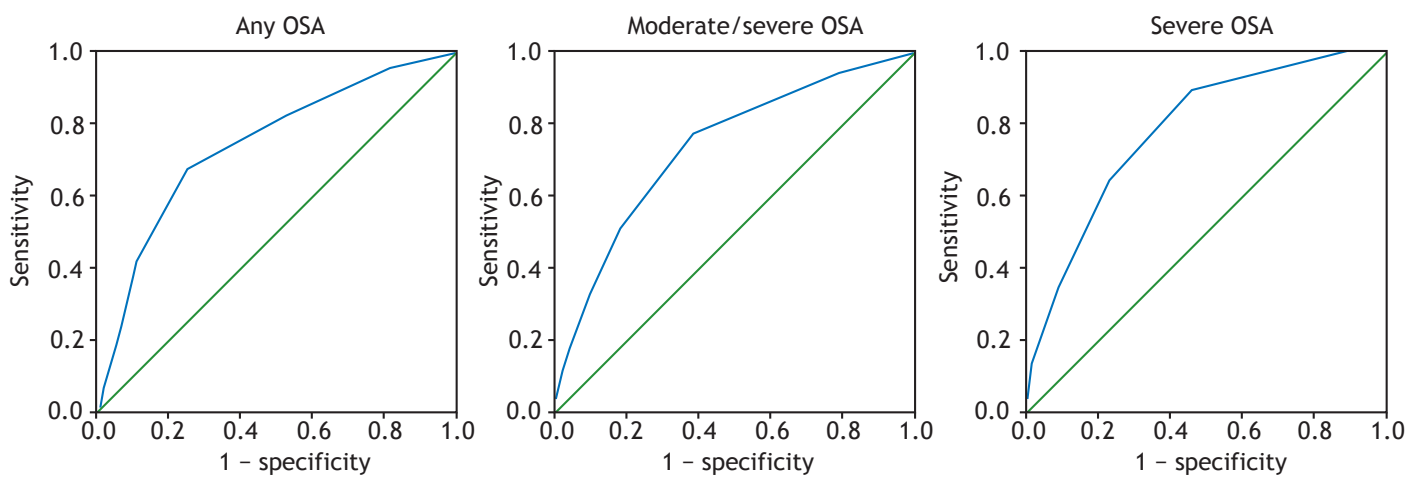

Figure 1. Graphic representation of all areas under the curve obtained for the Portuguese-language version of the STOP-Bang questionnaire, culturally adapted for use in Brazil $(n=456)$. Obstructive sleep apnea (OSA) was classified, based on the apnea-hypopnea index, as follows: $\geq 5$ events $/ \mathrm{h}=$ OSA (any degree); $\geq 15$ events $/ \mathrm{h}=$ moderate/severe OSA; and $\geq 30$ events $/ \mathrm{h}=$ severe OSA.

is higher in men than in women. ${ }^{(3,8,26-30)}$ In addition, men generally present with typical symptoms, such as snoring and observed apnea, whereas women are more likely to present with atypical symptoms, such as depression, fatigue, and insomnia. ${ }^{(26-29)}$ Anthropometric and demographic data also differ between genders: men generally have larger neck circumferences than do women, ${ }^{(31)}$ whereas women with OSA are generally older than their male counterparts. ${ }^{(3)}$ Furthermore, the performance of a questionnaire for OSA can vary widely depending on the population studied and the AHI cut-off point used for OSA diagnosis. ${ }^{(10,11)}$ The Berlin questionnaire, for example, was initially developed in a primary care setting, ${ }^{(13)}$ and its performance might therefore be better in that setting than in the sleep laboratory. ${ }^{(32)}$ In contrast, the STOP-Bang questionnaire 
Table 5. Predicting obstructive sleep apnea with the various possible scores on the Portuguese-language version of the STOP-Bang questionnaire $(n=456)$, by degree of severity. ${ }^{\mathrm{a}}$

\begin{tabular}{|c|c|c|c|c|c|c|}
\hline OSA severity & $\begin{array}{l}\text { High-risk } \\
\text { patients }^{b}\end{array}$ & $\begin{array}{l}\text { Low-risk } \\
\text { patients }^{b}\end{array}$ & OR & LR + & LR - & $\begin{array}{l}\text { Post-test } \\
\text { probability }\end{array}$ \\
\hline \multicolumn{7}{|l|}{$\mathrm{AHI} \geq 5$ events $/ \mathrm{h}$} \\
\hline$\geq 1$ vs. $<1$ & $454(99.6)$ & $2(0.4)$ & $3.63(0.09-134.11)$ & $1.00(0.99-1.02)$ & $0.27(0.00-10.11)$ & 78.3 \\
\hline$\geq 2$ vs. $<2$ & $423(92.8)$ & $33(7.2)$ & $5.06(2.31-11.12)$ & $1.17(1.07-1.27)$ & $0.23(0.11-0.46)$ & 80.8 \\
\hline$\geq 3$ vs. $<3$ & $352(77.2)$ & $104(22.8)$ & $4.20(2.52-7.03)$ & $1.53(1.28-1.85)$ & $0.36(0.26-0.51)$ & 84.7 \\
\hline$\geq 4$ vs. $<4$ & $267(58.6)$ & $189(41.4)$ & $6.22(3.65-10.66)$ & $2.68(1.92-3.91)$ & $0.43(0.36-0.52)$ & 90.6 \\
\hline$\geq 5$ vs. $<5$ & $161(35.3)$ & $295(64.7)$ & $5.79(2.88-11.91)$ & $3.78(2.13-7.17)$ & $0.65(0.60-0.73)$ & 93.2 \\
\hline$\geq 6$ vs. $<6$ & $79(17.3)$ & 377 (82.7) & $3.98(1.60-10.52)$ & $3.37(1.49-8.48)$ & $0.84(0.80-0.93)$ & 92.4 \\
\hline$\geq 7$ vs. $<7$ & $26(5.7)$ & $430(94.3)$ & $3.49(0.78-21.79)$ & $3.32(0.79-20.30)$ & $0.95(0.93-1.01)$ & 92.3 \\
\hline 8 vs. $<8$ & $4(0.9)$ & 452 (99.1) & $0.83(0.07-20.95)$ & $0.83(0.07-20.73)$ & $1.00(0.98-1.03)$ & 75.0 \\
\hline \multicolumn{7}{|l|}{$\mathrm{AHI} \geq 15$ events $/ \mathrm{h}$} \\
\hline$\geq 1$ vs. $<1$ & $454(99.6)$ & $2(0.4)$ & $\infty(0.26-\infty)$ & $1.00(0.99-1.00)$ & $0.00(0.00-3.73)$ & 52.0 \\
\hline$\geq 2$ vs. $<2$ & $423(92.8)$ & $33(7.2)$ & $4.42(1.78-11.47)$ & $1.10(1.04-1.14)$ & $0.24(0.10-0.58)$ & 54.4 \\
\hline$\geq 3$ vs. $<3$ & $352(77.2)$ & $104(22.8)$ & $4.21(2.52-7.07)$ & $1.36(1.23-1.49)$ & $0.32(0.21-0.48)$ & 59.6 \\
\hline$\geq 4$ vs. $<4$ & $267(58.6)$ & $189(41.4)$ & $5.21(3.40-8.00)$ & $1.97(1.66-2.34)$ & $0.37(0.29-0.48)$ & 68.1 \\
\hline$\geq 5$ vs. $<5$ & $161(35.3)$ & $295(64.7)$ & $4.45(2.85-6.96)$ & $2.70(1.99-3.72)$ & $0.60(0.53-0.69)$ & 74.5 \\
\hline$\geq 6$ vs. $<6$ & $79(17.3)$ & 377 (82.7) & $4.59(2.47-8.61)$ & $3.63(2.13-6.39)$ & $0.79(0.74-0.86)$ & 79.7 \\
\hline$\geq 7$ vs. $<7$ & $26(5.7)$ & $430(94.3)$ & $5.50(1.75-19.18)$ & $5.08(1.70-17.28)$ & $0.92(0.90-0.96)$ & 84.6 \\
\hline 8 vs. $<8$ & $4(0.9)$ & $452(99.1)$ & $2.79(0.25-70.25)$ & $2.77(0.26-69.10)$ & $0.99(0.98-1.01)$ & 75.0 \\
\hline \multicolumn{7}{|l|}{$\mathrm{AHI} \geq 30$ events $/ \mathrm{h}$} \\
\hline$\geq 1$ vs. $<1$ & $454(99.6)$ & $2(0.4)$ & $\infty(0.09-\infty)$ & $1.00(0.98-1.00)$ & $0.00(0.00-10.13)$ & 28.5 \\
\hline$\geq 2$ vs. $<2$ & $423(92.8)$ & $33(7.2)$ & $\infty(2.93-\infty)$ & $1.11(1.06-1.11)$ & $0.00(0.00-0.36)$ & 30.7 \\
\hline$\geq 3$ vs. $<3$ & $352(77.2)$ & $104(22.8)$ & $8.88(3.62-23.16)$ & $1.36(1.25-1.42)$ & $0.15(0.06-0.34)$ & 35.2 \\
\hline$\geq 4$ vs. $<4$ & $267(58.6)$ & $189(41.4)$ & $9.60(5.12-18.27)$ & $1.92(1.70-2.10)$ & $0.20(0.11-0.33)$ & 43.4 \\
\hline$\geq 5$ vs. $<5$ & $161(35.3)$ & $295(64.7)$ & $5.90(3.71-9.41)$ & $2.73(2.15-3.41)$ & $0.46(0.36-0.58)$ & 52.1 \\
\hline$\geq 6$ vs. $<6$ & $79(17.3)$ & 377 (82.7) & $5.20(3.02-8.96)$ & $3.68(2.42-5.61)$ & $0.70(0.62-0.80)$ & 59.5 \\
\hline$\geq 7$ vs. $<7$ & $26(5.7)$ & $430(94.3)$ & $7.80(3.00-21.05)$ & $6.80(2.78-17.54)$ & $0.87(0.83-0.93)$ & 73.0 \\
\hline 8 vs. $<8$ & $4(0.9)$ & $452(99.1)$ & $7.67(0.70-193.35)$ & $7.52(0.70-187.51)$ & $0.98(0.97-1.00)$ & 75.0 \\
\hline
\end{tabular}

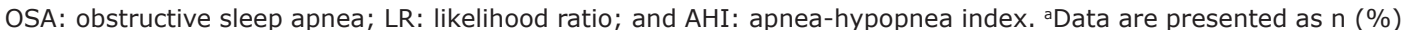

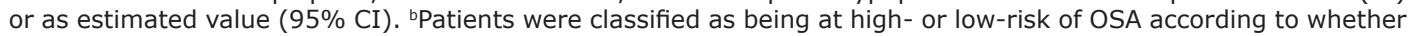
their score on STOP-Bang questionnaire was above or below the cut-off of 3 points, respectively.

was initially developed for use in surgical patients. ${ }^{(14)}$ Although the STOP-Bang questionnaire has been widely validated as a screening tool, ${ }^{(20)}$ it was found to be inadequate and inappropriate as a means of confirming the presence of significant OSA among patients at a Veterans Administration facility in the United States, possibly because of the low (4.9\%) specificity obtained in that study. ${ }^{(33)}$

The STOP-Bang is a screening instrument with high sensitivity, that sensitivity increasing in proportion to increases in the AHI threshold used (from 5 events/h to 30 events/h). ${ }^{(14)}$ Conversely, it exhibits low-to-moderate specificity, which reduces its accuracy. Because it prioritizes sensitivity over specificity, the STOP-Bang questionnaire classifies a large number of patients as high-risk, thus increasing the rate of false-positive results. Consequently, the STOP-Bang questionnaire alone is insufficient to rule out the need for a sleep study in all patients. Another important feature of the instrument is that an increase in its score (maximum, 8 points) has been shown to result in an increase in post-test probability. (34-36) Unfortunately, our study failed to show a linear increase in the post-test probability with increasing STOP-Bang scores, except in the patients with severe OSA, among whom such an increase was observed for all scores.

One previous study evaluated four tools for OSA screening ${ }^{(37)}$ : the four-variable screening tool, the STOP questionnaire, the STOP-Bang questionnaire, and the Epworth Sleepiness Scale. To predict moderate/severe OSA, the STOP-Bang questionnaire had the highest sensitivity $(87.0 \%)$, with an AUC of 0.64 , whereas the four-variable screening tool presented the highest specificity (93.2\%) and accuracy (79.4\%). Similar findings were reported in another study comparing five different questionnaires (the STOP questionnaire, the STOP-Bang questionnaire, the Berlin questionnaire, the Epworth Sleepiness Scale, and the four-variable screening tool).(38) In that study, the STOP-Bang questionnaire had the highest sensitivity (97.6\%), as well as the lowest specificity $(12.7 \%)$, for the identification of moderate/severe OSA.

The present study has some limitations that need to be emphasized. It involved patients referred to the sleep laboratory, who are typically pre-selected patients, which could represent a selection bias. In addition, we did not compare the performance of the STOP-Bang 
questionnaire with that of other validated screening instruments, and there is therefore a need for further studies comparing it with such tools. Nevertheless, our study was performed with a considerable sample of adults, all of whom underwent overnight polysomnography, which is the gold standard for the diagnosis of OSA. Additional strengths were the fact that it was a prospective study, with manual analysis of the polysomnography results, and the fact that the physicians involved in the polysomnography examinations had no prior knowledge of the STOP-Bang results.

In conclusion, the STOP-Bang questionnaire showed good performance in screening for OSA and can predict the severity of the disorder. The validation of the STOP-Bang questionnaire will promote its use as an important screening tool for OSA in sleep laboratories in Brazil. Because our study was conducted in a sleep laboratory setting, further studies are needed in order to validate the STOP-Bang questionnaire for use in other contexts, such as primary care.

\section{ACKNOWLEDGMENTS}

The authors would like to thank Dr. Frances Chung for her kindness in authorizing the validation of the STOP-Bang questionnaire for use in Brazil.

\section{REFERENCES}

1. Epstein LJ, Kristo D, Strollo PJ Jr, Friedman N, Malhotra A, Patil SP et al. Clinical guideline for the evaluation, management and longterm care of obstructive sleep apnea in adults. J Clin Sleep Med 2009;5(3):263-76.

2. Young T, Palta M, Dempsey J, Skatrud J, Weber S, Badr S. The occurrence of sleep-disordered breathing among middle-aged adults. N Engl J Med. 1993;328(17):1230-5. https://doi.org/10.1056/ NEJM199304293281704

3. Gabbay IE, Lavie P. Age- and gender-related characteristics of obstructive sleep apnea. Sleep Breath. 2012;16(2):453-60. https:// doi.org/10.1007/s11325-011-0523-z

4. Flegal KM, Kruszon-Moran D, Carroll MD, Fryar CD, Ogden CL. Trends in obesity among adults in the United States, 2005 to 2014. JAMA 2016;315(21):2284-91. https://doi.org/10.1001/jama.2016.6458

5. Tufik S, Santos-Silva R, Taddei JA, Bittencourt LR. Obstructive sleep apnea syndrome in the Sao Paulo Epidemiologic Sleep Study. Sleep Med. 2010;11(5):441-6. https://doi.org/10.1016/j.sleep.2009.10.005

6. Peppard PE, Young T, Barnet JH, Palta M, Hagen EW, Hla KM Increased prevalence of sleep-disordered breathing in adults. Am J Epidemiol. 2013;177(9):1006-14. https://doi.org/10.1093/aje/kws342

7. Heinzer $R$, Vat S, Marques-Vidal P, Marti-Soler H, Andries D, Tobback $\mathrm{N}$, et al. Prevalence of sleep-disordered breathing in the general population: the HypnoLaus study. Lancet Respir Med. 2015;3(4):310 8. https://doi.org/10.1016/S2213-2600(15)00043-0

8. Aurora RN, Collop NA, Jacobowitz O, Thomas SM, Quan SF, Aronsky AJ. Quality measures for the care of adult patients with obstructive sleep apnea. J Clin Sleep Med. 2015;11(3):357-83. https://doi. org/10.5664/jcsm.4556

9. Young T, Evans L, Finn L, Palta M. Estimation of the clinically diagnosed proportion of sleep apnea syndrome in middle-aged men and women. Sleep. 1997;20(9):705-6. https://doi.org/10.1093/ sleep/20.9.705

10. Abrishami A, Khajehdehi A, Chung F. A systematic review of screening questionnaires for obstructive sleep apnea. Can J Anesth 2010;57(5):423-38. https://doi.org/10.1007/s12630-010-9280-x

11. Ramachandran SK, Josephs LA. A meta-analysis of clinical screening tests for obstructive sleep apnea. Anesthesiology. 2009;110(4):92839. https://doi.org/10.1097/ALN.0b013e31819c47b6

12. Flemons WW, Whitelaw WA, Brant R, Remmers JE. Likelihood ratios for a sleep apnea clinical prediction rule. Am J Respir Crit Care Med. 1994;150(5 Pt 1):1279-85. https://doi.org/10.1164/ ajrccm.150.5.7952553

13. Netzer NC, Stoohs RA, Netzer CM, Clark K, Strohl KP. Using the Berlin Questionnaire to identify patients at risk for the sleep apnea syndrome. Ann Intern Med. 1999;131(7):485-91. https://doi. org/10.7326/0003-4819-131-7-199910050-00002

14. Chung $F$, Yegneswaran $B$, Liao $P$, Chung $S A$, Vairavanathan $S$, Islam $S$, et al. STOP questionnaire: a tool to screen patients for obstructive sleep apnea. Anesthesiology. 2008;108(5):812-21. https://doi. org/10.1097/ALN.0b013e31816d83e4

15. Takegami M, Hayashino Y, Chin K, Sokejima S, Kadotani H, Akashiba $T$, et al. Simple four-variable screening tool for identification of patients with sleep-disordered breathing. Sleep. 2009;32(7):939-48.

16. Marti-Soler H, Hirotsu C, Marques-Vidal P, Vollenweider P, Waeber G, Preisig $\mathrm{M}$, et al. The NoSAS score for screening of sleep-disordered breathing: a derivation and validation study. Lancet Respir Med.
2016:4(9):742-8. https://doi.org/10.1016/S2213-2600(16)30075-3

17. Fonseca LB, Silveira EA, Lima NM, Rabahi MF. STOP-Bang questionnaire: translation to Portuguese and cross-cultural adaptation for use in Brazil. J Bras Pneumol. 2016;42(4):266-72. https://doi. org/10.1590/s1806-37562015000000243

18. Berry RB, Budhiraja R, Gottlieb DJ, Gozal D, Iber C, Kapur VK, et al. Rules for scoring respiratory events in sleep: update of the 2007 AASM Manual for the Scoring of Sleep and Associated Events. Deliberations of the Sleep Apnea Definitions Task Force of the American Academy of Sleep Medicine. J Clin Sleep Med. 2012;8(5):597-619.

19. Singh M, Liao P, Kobah S, Wijeysundera DN, Shapiro C, Chung F Proportion of surgical patients with undiagnosed obstructive sleep apnoea. Br J Anaesth. 2013;110(4):629-36. https://doi.org/10.1093/ bja/aes465

20. Nagappa M, Liao P, Wong J, Auckley D, Ramachandran SK Memtsoudis S, et al. Validation of the STOP-Bang questionnaire as a screening tool for obstructive sleep apnea among different populations: a systematic review and meta-analysis. PLoS One. 2015;10(12):e0143697. https://doi.org/10.1371/journal. pone.0143697

21. Luo J, Huang R, Zhong $X$, Xiao $Y$, Zhou J. Value of STOP-Bang questionnaire in screening patients with obstructive sleep apnea hypopnea syndrome in sleep disordered breathing clinic. Chin Med $J$ (Engl). 2014;127(10):1843-8

22. Bille DJ, Bille-Hasselstrøm C, Petersen CG. Translation and validation of the Stop-Bang Questionnaire for obstructive sleep apnoea into Danish. Dan Med J. 2015;62(12):A5158.

23. Sadeghniiat-Haghighi K, Montazeri A, Khajeh-Mehrizi A, Ghajarzadeh $\mathrm{M}$, Alemohammad ZB, Aminian $\mathrm{O}$, et al. The STOP-BANG questionnaire: reliability and validity of the Persian version in sleep clinic population. Qual Life Res. 2015;24(8):2025-30. https://doi. org/10.1007/s11136-015-0923-9

24. Alhouqani S, Al Manhali M, Al Essa A, Al-Houqani M. Evaluation of the Arabic version of STOP-Bang questionnaire as a screening too for obstructive sleep apnea. Sleep Breath. 2015;19(4):1235-40. https://doi.org/10.1007/s11325-015-1150-x

25. Reis R, Teixeira F, Martins V, Sousa L, Batata L, Santos C, et al. Validation of a Portuguese version of the STOP-Bang questionnaire as a screening tool for obstructive sleep apnea: Analysis in a sleep clinic. Rev Port Pneumol (2006). 2015;21(2):61-8. https://doi. org/10.1016/.j.rppnen.2014.04.009

26. Quintana-Gallego E, Carmona-Bernal C, Capote F, Sánchez-Armengo A, Botebol-Benhamou G, Polo-Padillo J, et al. Gender differences in obstructive sleep apnea syndrome: a clinical study of 1166 patients. Respir Med. 2004;98(10):984-9. https://doi.org/10.1016/j. rmed.2004.03.002

27. Basoglu OK, Tasbakan MS. Gender differences in clinical and polysomnographic features of obstructive sleep apnea: a clinica study of 2827 patients. Sleep Breath. 2017 Feb 14 [Epub ahead of print] https://doi.org/10.1007/s11325-017-1482-9

28. Valipour $A$, Lothaller $H$, Rauscher $H$, Zwick H, Burghuber OC, Lavie P. Gender-related differences in symptoms of patients with suspected breathing disorders in sleep: a clinical population study using the sleep disorders questionnaire. Sleep. 2007;30(3):312-9. https://doi. org/10.1093/sleep/30.3.312 
29. Shah N, Hanna DB, Teng Y, Sotres-Alvarez D, Hall M, Loredo JS, et al. Sex-specific prediction models for sleep apnea from the Hispanic Community Health Study/Study of Latinos. Chest. 2016;149(6):140918. https://doi.org/10.1016/j.chest.2016.01.013

30. Duarte RL, Magalhães-da-Silveira FJ. Factors predictive of obstructive sleep apnea in patients undergoing pre-operative evaluation for bariatric surgery and referred to a sleep laboratory for polysomnography. J Bras Pneumol. 2015;41(5):440-8. https://doi. org/10.1590/S1806-37132015000000027

31. Dancey DR, Hanly PJ, Soong C, Lee B, Shepard J Jr, Hoffstein $\checkmark$. Gender differences in sleep apnea: the role of neck circumference. Chest 2003;123(5):1544-50. https://doi.org/10.1378/ chest.123.5.1544

32. Ahmadi N, Chung SA, Gibbs A, Shapiro CM. The Berlin questionnaire for sleep apnea in a sleep clinic population: relationship to polysomnographic measurement of respiratory disturbance. Sleep Breath. 2008;12(1):39-45. https://doi.org/10.1007/s11325-007-0125-y

33. Kunisaki KM, Brown KE, Fabbrini $A E$, Wetherbee $E E$, Rector TS. STOP-BANG questionnaire performance in a Veterans Affairs unattended sleep study program. Ann Am Thorac Soc. 2014;11(2):192-7. https://doi.org/10.1513/AnnalsATS.201305-134OC
34. Chung F, Subramanyam R, Liao P, Sasaki E, Shapiro C, Sun Y. High STOP-Bang score indicates a high probability of obstructive sleep apnoea. Br J Anaesth. 2012;108(5):768-75. https://doi.org/10.1093/ bja/aes022

35. Chung F, Yang Y, Liao P. Predictive performance of the STOP-Bang score for identifying obstructive sleep apnea in obese patients. Obes Surg. 2013;23(12):2050-7. https://doi.org/10.1007/s11695-0131006-z

36. Farney RJ, Walker BS, Farney RM, Snow GL, Walker JM. The STOPBang equivalent model and prediction of severity of obstructive sleep apnea: relation to polysomnographic measurements of the apnea/ hypopnea index. J Clin Sleep Med. 2011;7(5):459-65B. https://doi. org/10.5664/jcsm. 1306

37. Silva GE, Vana KD, Goodwin JL, Sherrill DL, Quan SF. Identification of patients with sleep disordered breathing: comparing the four-variable screening tool, STOP, STOP-Bang, and Epworth Sleepiness Scales. J Clin Sleep Med. 2011;7(5):467-72. https://doi.org/10.5664/jcsm.1308

38. Pataka A, Daskalopoulou E, Kalamaras G, Fekete Passa K Argyropoulou P. Evaluation of five different questionnaires for assessing sleep apnea syndrome in a sleep clinic. Sleep Med. 2014;15(7):776-81. https://doi.org/10.1016/..sleep.2014.03.012 